\title{
Facial Nerve Outcome after Vestibular Schwannoma Resection: A Comparative Meta-Analysis of Endoscopic versus Open Retrosigmoid Approach
}

\author{
Abdullah Alobaid ${ }^{1}$ Mohammed Aref ${ }^{1}$ Michael Ross Bennardo ${ }^{1} \quad$ Forough Farrokhyar $^{2}$ Kesava Reddy ${ }^{1}$ \\ ${ }^{1}$ Division of Neurosurgery, McMaster University, Hamilton, \\ Ontario, Canada \\ 2 Department of Surgery, McMaster University, Hamilton, \\ Ontario, Canada \\ Address for correspondence Abdullah Alobaid, MD, FRCSC, 96 St. \\ Patrick Street, Apt. 1210, Toronto, Ontario M5T1V2, Canada \\ (e-mail: abdullah.alobaid@medportal.ca).
}

J Neurol Surg B 2015;76:157-162.

\begin{abstract}
Keywords

- vestibular schwannoma

- endoscopic surgery

- retrosigmoid approach

- facial nerve

The minimal access retrosigmoid endoscopic approach to vestibular schwannoma (VS) resection has been used with promising results. However, it has not been compared with the standard open approach in the literature. We performed a meta-analysis review for all articles describing both approaches for VS from 1996 to 2011. We found 1861 articles. After review and discussion, we narrowed our study to 25 articles, 4 endoscopic and 21 open. The total number of patients was 3026 for open and 790 for endoscopic. The mean tumor sizes in the open and endoscopic series were $2.5 \mathrm{~cm}$ and $2.7 \mathrm{~cm}$, respectively. Good facial nerve outcome was achieved in $67 \%$ of the open series patients and in $94 \%$ of the endoscopic series patients. Other outcomes in the open and endoscopic series were the following: gross total resection, $91 \%$ versus $97 \%$; functional hearing, $22.6 \%$ versus $46 \%$; wound infection, $1.3 \%$ versus $2.6 \%$; and recurrence, $5.4 \%$ versus $2.2 \%$. We acknowledge the limitations of our study, but we can state that the endoscopic approach is not inferior to the standard open approach. In expert hands the endoscopic approach can offer as good a result as the open, with potential benefits such as less pain and a shorter length of stay in the hospital. There is a need for more controlled studies for a definitive comparison.
\end{abstract}

\section{Introduction}

Vestibular schwannoma (VS) is a benign nerve sheath tumor arising most commonly from the superior vestibular nerve, at the entrance to the internal acoustic meatus. According to the National Institutes of Health, 2000 to 3000 cases are diagnosed per year in the United States and represent 6 to $10 \%$ of all primary brain tumors, and 70 to $90 \%$ of all cerebellopontine angle (CPA) tumors. The first successful removal of VS was probably performed by Sir Charles Balance in 1894. During the last few decades, the remarkable improvement in tech-

received

April 5, 2014

accepted

May 6, 2014

published online

November 26, 2014

nology has shifted the focus from survival to facial nerve preservation, preservation of serviceable hearing, and gross total resection (GTR) of the tumor. The three classic approaches to VS are retrosigmoid, translabyrinthine, and middle fossa. Of these approaches, the retrosigmoid is the most popular among the neurosurgical community. Endoscopy is relatively novel and a rapidly growing surgical advance that provides excellent visualization with minimal access. This has resulted in a transformation of open surgical approaches to minimally invasive approaches. The best example is transsphenoidal approaches for lesions of the sella and skull base.

C 2015 Georg Thieme Verlag KG Stuttgart · New York
DOI http://dx.doi.org/ 10.1055/s-0034-1383858. ISSN 2193-6331. 
Some surgical groups have adapted the minimally invasive technique for VS, specifically the minimal access endoscopic retrosigmoid approach, with promising results. However, there has been no study reported in the literature comparing the minimally invasive and the standard open retrosigmoid approach.

Our goal was to determine whether patient outcomes and complications differ between the two surgical approaches for the removal of VS: minimal access endoscopic or open retrosigmoid resection.

\section{Methods}

The primary outcome is the difference in the rate of facial nerve preservation between the two approaches with this null hypothesis: "there is no difference between the two surgical modalities with regard to facial nerve preservation." Secondary outcomes include rates of hearing preservation, GTR, cerebrospinal fluid (CSF) leak, wound infection, recurrence, and death. All these outcomes were collected for metaanalysis. The mean tumor size in each of the two groups were also collected and put into the analysis to get a better comparison. We explored the possibility of addressing other outcomes such as hospital length of stay (LOS) but were unable to do so due to inconsistent reporting. We performed a thorough search on the and Ovid databases for all studies about VS, without any other limiting keywords, from 1996 to 2011. For endoscopic series, we also used the references for each article, due to the limited number of studies in the literature. The inclusion and exclusion criteria were established a priori and are as follows.

\section{Inclusion Criteria}

- Case series with $\geq 20$ patients. This sample size was suggested by the senior author, to get the most expert and higher volume cases series.

- Utilization of endoscope or microscope for resection.

- Retrosigmoid incision and craniotomy.

- Adult patients.

- English-language publication.

\section{Exclusion Criteria}

- Studies describing other open approaches (i.e., translabyrinthine, middle fossa).

- Animal, cadaver, and basic science studies.

- Studies not addressing the primary outcome (i.e., facial nerve function).

- Comparative studies due to difficulty to extract accurate data.

We use the House-Brackmann (HB) classification for facial nerve outcome. ${ }^{1}$ For ease of analysis, we combined grades 1 and 2 and labeled these as good, 3 and 4 as moderate, and 5 and 6 as poor. With regard to hearing outcome, ${ }^{2}$ we include "serviceable" and "some" (i.e., pure tone audiometry [PTA] $<80 \mathrm{~dB}$ and speech discrimination $>20 \%$ ); in one group, we call it functional or meaningful hearing. Two independent observers (A.A. and M.A.) reviewed all articles, first by title and abstract and then the entire article if needed. We assumed between-study heterogeneity due to the limitations inherent in the nature of the study design (case series). Percentages with 95\% confidence intervals (CIs) are reported. StatsDirect software (StatsDirect.com, Altrincham, United Kingdom) was used for the calculations.

\section{Results}

We found 1861 articles. Both observers (A.A. and M.A.) excluded 1730 articles, and both of them included 44 articles. Disagreements were resolved by discussion among the two reviewers with advice from the senior author (K.R.). Finally we included 25 articles: 4 endoscopic and 21 open ( - Table 1 ). The total population of patients was 3026 for the open approach and 790 for the endoscopic approach. The mean maximum tumor diameters in the open and endoscopic series were $2.5 \mathrm{~cm}$ and 2.7, respectively. - Table 2 summarizes the results of the meta-analysis with $95 \%$ CIs. A higher rate of good facial nerve outcome appeared to be observed in the endoscopic series (94\% versus 67\%) (-Figs. 1 and 2). Higher rates of GTR and meaningful hearing also seemed to be achieved in the endoscopic series (97\% versus $91 \%$, and $46 \%$ versus $22.6 \%$, respectively) (-Figs. 3 and $\mathbf{4}$ ). With regard to negative outcomes, the endoscopic retrosigmoid approach seemed to have a lower rate of CSF leak (4.6\% versus $8.2 \%$ ) and recurrence $(2.6 \%$ versus $5.4 \%$ ) but a higher rate of wound infections ( $2.6 \%$ versus $1.3 \%$ ). The difference in mortality rate was minimal.

\section{Discussion}

To the best of our knowledge, the first publication utilizing endoscope for VS surgery was by Valtonen et al in $1997 .^{3}$ The authors used an endoscope to visualize the deep-seated air cells, after drilling the posterior wall of the internal acoustic meatus (IAM), in an effort to decrease the CSF leak rate after microscopic resection. Since the initial publication, a small but growing number of surgeons have reported their experiences using the endoscope as an adjunct to the surgical microscope and microsurgical techniques. Shahinian and colleagues were the first to describe a fully endoscopic approach to the VS 4 in 2004.

To date, the biggest series on fully endoscopic minimal access retrosigmoid approach to VS was conducted by Shahinian et $\mathrm{al}^{5}$ in 2011. The patient population consisted of 527 cases with unilateral VS; patients with neurofibromatosis type 2 were excluded. Tumors ranged from 0.3 to $5.8 \mathrm{~cm}$; most tumors were $<4 \mathrm{~cm}$ in diameter (mean: $2.8 \mathrm{~cm}$ ). Tumors were removed via $2.0-\mathrm{cm}$ retrosigmoid craniotomies. All cases utilize electromyographic facial nerve monitoring. The completeness of tumor removal was judged by surgical records (intraoperative digital pictures) and postoperative magnetic resonance imaging. GTR was achieved in $94 \%$ of the cases. Anatomical preservation of the facial nerve was achieved in $100 \%$ of the cases, as judged by the operating 
Table 1 Final studies included in the analysis

\begin{tabular}{|c|c|c|}
\hline & Study & $\begin{array}{l}\text { No. of patients } \\
\text { included in } \\
\text { final analysis }\end{array}$ \\
\hline \multirow{21}{*}{$\begin{array}{l}\text { Open } \\
\text { approach }\end{array}$} & Samii et $\mathrm{al}^{7-10}$ & 962 \\
\hline & Colletti and Fiorino ${ }^{12}$ & 103 \\
\hline & Moffat et al ${ }^{13}$ & 50 \\
\hline & Jung et $\mathrm{al}^{14}$ & 30 \\
\hline & Mazzoni et al ${ }^{15}$ & 150 \\
\hline & Tonn et $\mathrm{al}^{16}$ & 508 \\
\hline & Strauss $^{17}$ & 22 \\
\hline & Lassaletta et al $^{18}$ & 65 \\
\hline & Maw et al $^{19}$ & 40 \\
\hline & Yamakami et $a^{20}$ & 50 \\
\hline & Darwish et $\mathrm{al}^{21}$ & 97 \\
\hline & Zhang et $\mathrm{al}^{22}$ & 105 \\
\hline & Samii et $\mathrm{al}^{23}$ & 200 \\
\hline & Sinha and Sharma ${ }^{24}$ & 58 \\
\hline & Veronezi et $\mathrm{al}^{25}$ & 20 \\
\hline & Yang et al $^{11}$ & 110 \\
\hline & Chen et $\mathrm{al}^{26}$ & 103 \\
\hline & Chen et $\mathrm{al}^{27}$ & 145 \\
\hline & Zhao et $\mathrm{al}^{28}$ & 89 \\
\hline & Di Maio et al $^{29}$ & 47 \\
\hline & Gerganov et $\mathrm{al}^{30}$ & 53 \\
\hline \multirow{4}{*}{$\begin{array}{l}\text { Endoscopic } \\
\text { approach }\end{array}$} & Göksu et al ${ }^{31}$ & 32 \\
\hline & Magnan et $\mathrm{al}^{32}$ & 119 \\
\hline & Kabil and Shahinian 33 & 112 \\
\hline & Shahinian and $\mathrm{Ra}^{5}$ & 527 \\
\hline
\end{tabular}

surgeon. One year after tumor removal, facial nerve function was reassessed. Of 527 patients with anatomically preserved facial nerves, $93 \%$ showed good facial nerve function (HB grade 1 or 2 ); $4 \%$ showed moderate function (HB grade 3 or 4 ); and $3 \%$ showed poor function (HB grade 5 or 6 ). Preoperatively, there were 374 patients with functional hearing, defined as a PTA $<80 \mathrm{~dB}$ and speech discrimination $>20 \%$. Postoperatively, 213 of those 374 (57\%) maintained or gained better hearing function. The length of stay (LOS) ranged from 1 to 6 days (mean: 2.4 days).

Another endoscopic series worth mentioning that was not available at the time of analysis was conducted by Pieper and colleagues. ${ }^{6}$ Over 5 years, their center performed 147 endoscopic posterolateral approaches: 110 procedures for cranial nerve microvascular decompressions and neurectomies and 37 for tumor resections, 23 specifically for VS tumors. For tumor cases, they used dissectors that provide constant stimulation to monitor the facial nerve while removing the tumors. There was a clinically significant decline in hearing in three patients (8\%); all of them occurred in VS tumors (13\%). There were no occurrences of facial nerve weakness in any of the VS patients. There was one case of a delayed CSF leak that was repaired surgically. Two wound infections required removal of the hydroxyapatite cement. Most patients are in the hospital for 24 to 72 hours. Those patients who did not experience any vertigo postoperatively were able to return to work within 2 weeks of surgery.

We have summarized the open series data ( - Table 2 ), but we will describe two of the studies in some detail, one prior to the 2000 and the other one after 2000 . Over almost 2 decades, 1000 VSs were removed from 962 patients using the open retrosigmoid approach by Samii et al. ${ }^{7-10}$ of the $1000 \mathrm{VS}$ cases, in 979 (97.9\%) GTR was achieved. The facial nerve was anatomically preserved in 929 cases (93\%). Facial nerve function, graded according to the HB scale within 2 weeks after surgery, was grade 1 in $47 \%$, grade 2 in $12 \%$, grade 3 in $14 \%$, grade 4 in $6 \%$, grade 5 in $10 \%$, and grade 6 in $11 \%$ of the patients. The $11 \%$ with grade 6 included $7 \%$ of the cases of facial nerve discontinuity and $4 \%$ of paralysis despite nerve continuity. Most of the patients with paralysis despite nerve continuity showed recovery within the first postoperative year. Of the $1.7 \%$ who did not improve at 10 to 12 months, 11 patients were treated by reanimation procedures, and a few underwent plastic surgery because they refused nerve reconstruction. So in summary, of patients with anatomical

Table 2 Summary of meta-analysis comparing open series with minimal access endoscopic series with 95\% confidence interval

\begin{tabular}{|l|l|l|}
\hline & $\begin{array}{l}\text { Open retrosigmoid, } \\
\%(95 \% \mathrm{Cl})\end{array}$ & $\begin{array}{l}\text { Minimal access endoscopic } \\
\text { retrosigmoid, \% (95\% CI) }\end{array}$ \\
\hline Good facial nerve outcome (HB 1 or 2) & $67.0 \%(61-73 \%)$ & $94 \%(92-95 \%)$ \\
\hline GTR & $91 \%(80-98 \%)$ & $97 \%(92-99 \%)$ \\
\hline $\begin{array}{l}\text { Meaningful hearing (PTA }<80 \mathrm{~dB} \text { and } \\
\text { speech discrimination }>20 \%)\end{array}$ & $22.6 \%(10.4-37.6 \%)$ & $46 \%(38-54 \%)$ \\
\hline CSF leak & $8.2 \%(4.8-12.3 \%)$ & $4.6 \%(2.3-7.2 \%)$ \\
\hline Wound infection & $1.3 \%(0.6-2.3 \%)$ & $2.6 \%(1.5-4.0 \%)$ \\
\hline Recurrence & $5.4 \%(1.8-10.1 \%)$ & $2.2 \%(1.3-3.4 \%)$ \\
\hline Death & $0.9 \%(0.3-2 \%)$ & $0 \%$ \\
\hline
\end{tabular}

Abbreviations: CSF, cerebrospinal fluid; GTR, gross total resection; HB, House-Brackmann (scale); PTA, pure tone audiometry. 


\section{Proportion meta-analysis plot [random effects]}

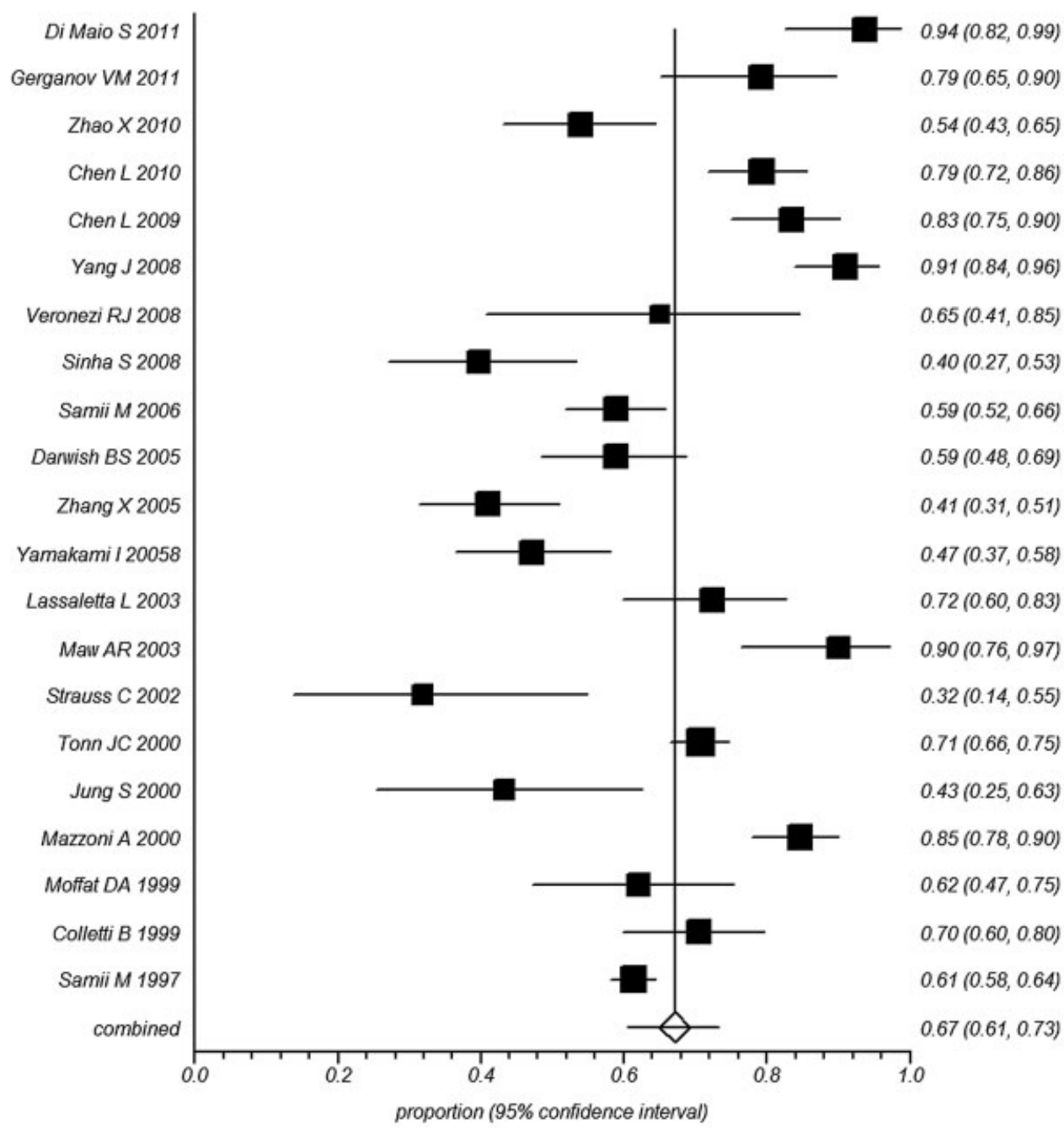

Fig. 1 Forest plot for good facial nerve outcome (House-Brackmann scale 1 or 2 ) in the open series.

preservation, $64 \%$ had good facial nerve function (HB 1 or 2 ) well documented in the 2 - to 8 -week postoperative period. In the final 200 cases, there was more preservation of the facial nerve (likely due to more frequent use of neurophysiologic

$$
\text { Proportion meta-analysis plot [random effects] }
$$

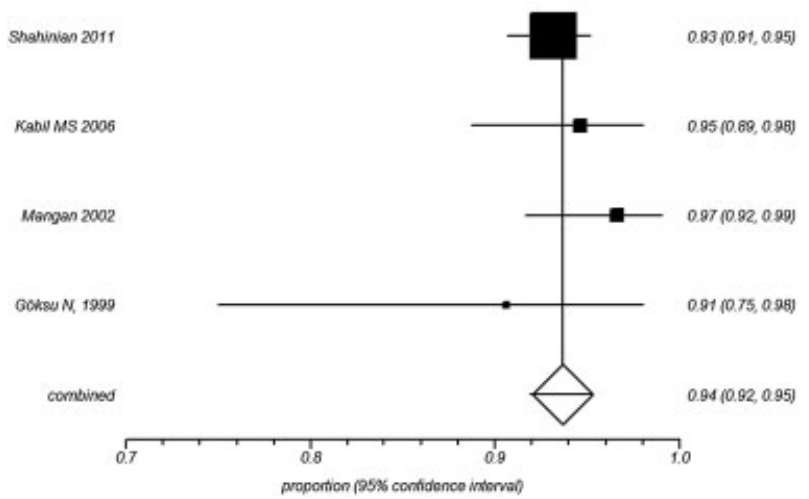

Fig. 2 Forest plot of good facial nerve outcome (House-Brackmann scale 1 or 2 ) in the endoscopic series. monitoring). Larger and cystic tumors appeared to have a lower rate of facial preservation. With regard to hearing, of a total of 732 cases with some preoperative hearing, anatomical cochlear nerve preservation was achieved in 580 cases (79\%)

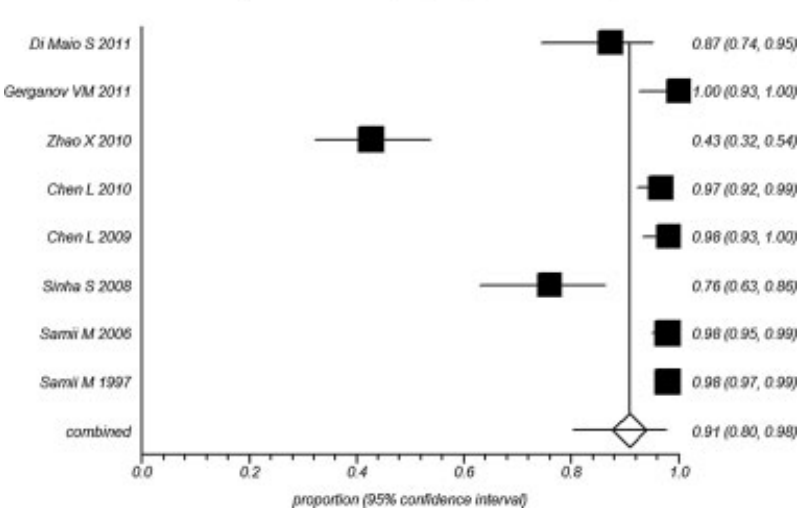

Fig. 3 Forest plot for gross total resection rate in the open series. 


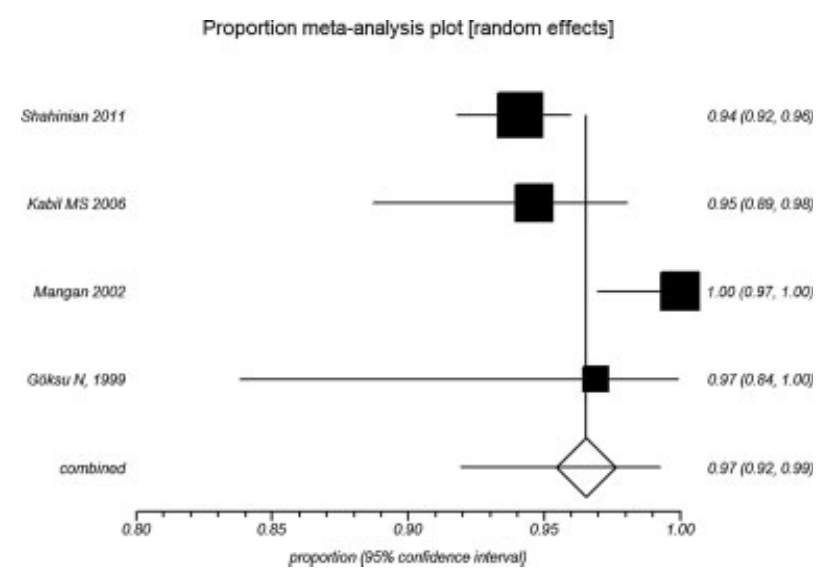

Fig. 4 Forest plot for gross total resection rate in the endoscopic series.

and functional cochlear nerve preservation in 289 (39.5\%); analysis over time revealed an actual preservation rate of $47 \%$ in the most recent 200 cases. The LOS in the hospital ranged from 8 to 14 days.

In 2008, Yang et al ${ }^{11}$ presented their experience with 110 open retrosigmoid approaches for VS. The mean tumor size (including the intracanalicular portion of the tumor) was $15 \mathrm{~mm}$ (range: $8-25 \mathrm{~mm}$ ). The preservation of a good facial function (HB grade 1 or 2 ) was achieved in $91 \%$ of patients at 1- to 2-year follow-up after surgery. Among patients with a preoperative class A or B in the American Academy of Otolaryngology-Head and Neck Surgery, AAO-HNS (99 patients), a postoperative useful hearing (classes A, B, and C) was achieved in $44 \%$. Hearing preservation appeared to be influenced by tumor size and preoperative hearing status.

In our study with specific inclusion and exclusion criteria and outcomes that were set forth a priori, we examined the literature systematically and attempted to extract raw data to achieve the best possible comparison between the two surgical techniques. Because there were no studies directly comparing the open retrosigmoid with the endoscopic retrosigmoid approach, the classic meta-analysis was not possible, thus resulting in a systematic review based on available raw data from each group.

There seemed to be better facial nerve preservation in the endoscopic series. Given the significant limitations of this study, we can only speculate about the causes. Two possible causes may be considered. First, the vast majority of endoscopic series are relatively new, so the improved facial nerve outcome may be attributable to early detection, improvement of surgical technique in general, and better availability of intraoperative neurophysiologic monitoring. Two other authors ${ }^{5,6}$ stated that by using an endoscope (including a rigid angled endoscope), better visualization of CPA structures can be achieved with early visualization of the facial nerve course in its entirety. These speculations may also be applied to explain the better hearing preservation and GTR found in our analysis. The lower CSF leak rate is probably due to better visualization that helps to locate and block the deep-seated air cells after drilling the IAM. ${ }^{3}$ However, there was a higher infection rate in the endoscopic series, which could be due to variability in reporting the infection rates in the literature. Although it was not included in our analysis, there seemed to be a tendency to a shorter LOS in the endoscopic series. These data were not consistently extractable from the studies to perform any valid comparisons. That could be due to a smaller craniotomy and minimal cerebellar retraction in the endoscopic cases. ${ }^{5}$

We acknowledge the following limitation in our study:

- The significant heterogeneity in between retrospective studies

- Poor availability of comparable data

- Different definitions and scales, particularly for audition

- Crossover of patient population between some studies

- The bulk of the endoscopic cases came from one surgeon's series $^{5}$

- Significant difference in the size of the groups and the fact that the two groups were really performed at different time periods so the open series are disadvantaged in this analysis

\section{Conclusion}

We have performed a systematic analysis of available data and must acknowledge its serious limitations. The results seem to suggest that the minimal access endoscopic retrosigmoid approach is not inferior to the standard open approach. It appears that in the hands of an expert, the endoscopic approach to VS may offer results as good as the open approach with potential benefits including better pain control, shorter LOS, and other possible outcomes. There is a significant need for better experience with the endoscopic approach from different parts of the world that may enable better comparative studies.

\section{References}

1 House JW, Brackmann DE. Facial nerve grading system. Head Neck Surg 1985;93:146-147

2 Jarrahy R, Eby JB, Cha ST, Shahinian HK. Fully endoscopic vascular decompression of the trigeminal nerve. Minim Invasive Neurosurg 2002;45(1):32-35

3 Valtonen HJ, Poe DS, Heilman CB, Tarlov EC. Endoscopically assisted prevention of cerebrospinal fluid leak in suboccipital acoustic neuroma surgery. Am J Otol 1997;18(3):381-385

4 Shahinian HK, Eby JB, Ocon M. Fully endoscopic excision of vestibular schwannomas. Minim Invasive Neurosurg 2004;47(6): 329-332

5 Shahinian HK, Ra Y. 527 fully endoscopic resections of vestibular schwannomas. Minim Invasive Neurosurg 2011;54(2):61-67

6 Pieper DR. The endoscopic approach to vestibular schwannomas and posterolateral skull base pathology. Otolaryngol Clin North Am 2012;45(2):439-454, x x.

7 Matthies C, Samii M. Management of 1000 vestibular schwannomas (acoustic neuromas): clinical presentation. Neurosurgery 1997;40(1):1-9; discussion 9-10

8 Samii M, Matthies C. Management of 1000 vestibular schwannomas (acoustic neuromas): the facial nerve-preservation and restitution of function. Neurosurgery 1997;40(4):684-694; discussion 694-695 
9 Samii M, Matthies C. Management of 1000 vestibular schwannomas (acoustic neuromas): hearing function in 1000 tumor resections. Neurosurgery 1997;40(2):248-260; discussion 260-262

10 Samii M, Matthies C. Management of 1000 vestibular schwannomas (acoustic neuromas): surgical management and results with an emphasis on complications and how to avoid them. Neurosurgery 1997;40(1):11-21, discussion 21-23

11 Yang J, Grayeli AB, Barylyak R, Elgarem H. Functional outcome of retrosigmoid approach in vestibular schwannoma surgery. Acta Otolaryngol 2008;128(8):881-886

12 Colletti V, Fiorino FG. Retrosigmoid-transmeatal en bloc removal of small to medium-sized acoustic neuromas. Otolaryngologyhead and neck surgery: official journal of American Academy of Otolaryngology-. Head Neck Surg 1999;120:122-128

13 Moffat DA, da Cruz MJ, Baguley DM, Beynon GJ, Hardy DG. Hearing preservation in solitary vestibular schwannoma surgery using the retrosigmoid approach. . Head Neck Surg 1999;121:781-788

14 Jung S, Kang SS, Kim TS, et al. Current surgical results of retrosigmoid approach in extralarge vestibular schwannomas. Surg Neurol 2000;53(4):370-377; discussion 377-378

15 Mazzoni A, Calabrese V, Danesi G. A modified retrosigmoid approach for direct exposure of the fundus of the internal auditory canal for hearing preservation in acoustic neuroma surgery. Am J Otol 2000;21(1):98-109

16 Tonn JC, Schlake HP, Goldbrunner R, Milewski C, Helms J, Roosen K. Acoustic neuroma surgery as an interdisciplinary approach: a neurosurgical series of 508 patients. J Neurol Neurosurg Psychiatry 2000;69(2):161-166

17 Strauss $C$. The facial nerve in medial acoustic neuromas. J Neurosurg 2002;97(5):1083-1090

18 Lassaletta L, Fontes L, Melcon E, Sarria MJ, Gavilan J. Hearing preservation with the retrosigmoid approach for vestibular schwannoma: myth or reality? . Head Neck Surg 2003;129:397-401

19 Maw AR, Coakham HB, Ayoub O, Butler SR. Hearing preservation and facial nerve function in vestibular schwannoma surgery. Clin Otolaryngol Allied Sci 2003;28(3):252-256

20 Yamakami I, Uchino Y, Kobayashi E, Yamaura A, Oka N. Removal of large acoustic neurinomas (vestibular schwannomas) by the retrosigmoid approach with no mortality and minimal morbidity. J Neurol Neurosurg Psychiatry 2004;75(3):453-458

21 Darwish BS, Bird PA, Goodisson DW, Bonkowski JA, MacFarlane MR. Facial nerve function and hearing preservation after retrosigmoid excision of vestibular schwannoma: Christchurch Hospital experience with 97 patients. ANZ J Surg 2005;75(10):893-896

22 Zhang X, Fei Z, Chen YJ, et al. Facial nerve function after excision of large acoustic neuromas via the suboccipital retrosigmoid approach. J Clin Neurosci 2005;12(4):405-408

23 Samii M, Gerganov V, Samii A. Improved preservation of hearing and facial nerve function in vestibular schwannoma surgery via the retrosigmoid approach in a series of 200 patients. J Neurosurg 2006;105(4):527-535

24 Sinha S, Sharma BS. Cystic acoustic neuromas: surgical outcome in a series of 58 patients. J Clin Neurosci 2008;15(5):511-515

25 Veronezi RJ, Fernandes YB, Borges G, Ramina R. Long-term facial nerve clinical evaluation following vestibular schwannoma surgery. Arq Neuropsiquiatr 2008;66 0A 194-198

26 Chen L, Chen L, Liu L, et al. Vestibular schwannoma microsurgery with special reference to facial nerve preservation. Clin Neurol Neurosurg 2009;111(1):47-53

27 Chen L, Chen LH, Ling F, Liu YS, Samii M, Samii A. Removal of vestibular schwannoma and facial nerve preservation using small suboccipital retrosigmoid craniotomy. Chin Med J (Engl) 2010; 123(3):274-280

28 Zhao X, Wang Z, Ji Y, et al. Long-term facial nerve function evaluation following surgery for large acoustic neuromas via retrosigmoid transmeatal approach. Acta Neurochir (Wien) 2010;152(10):1647-1652

29 Di Maio S, Malebranche AD, Westerberg B, Akagami R. Hearing preservation after microsurgical resection of large vestibular schwannomas. Neurosurgery 2011;68(3):632-640; discussion 640

30 Gerganov VM, Pirayesh A, Nouri M, et al. Hydrocephalus associated with vestibular schwannomas: management options and factors predicting the outcome. J Neurosurg 2011;114(5): 1209-1215

31 Göksu N, Bayazit Y, Kemaloğlu Y. Endoscopy of the posterior fossa and dissection of acoustic neuroma. J Neurosurg 1999;91(5): 776-780

32 Magnan J, Barbieri M, Mora R, et al. Retrosigmoid approach for small and medium-sized acoustic neuromas. Otol Neurotol 2002; 23(2):141-145

33 Kabil MS, Shahinian HK. A series of 112 fully endoscopic resections of vestibular schwannomas. Minim Invasive Neurosurg 2006; 49(6):362-368 